\title{
QPFORSETI
}

Forseti. Revista de Derecho. Volumen 8, № 11, Lima, 2020, pp. 149 - 166

\section{Tendiendo puentes entre la ingeniería y el derecho: El Building Information Modelling (BIM) y su impacto en la obra pública}

\author{
Leandro García Valdez*
}

Resumen. - En el presente artículo, el autor analiza la importancia del Building Information Modelling (BIM) y sus beneficios para combatir la rigidez normativa con respecto a la obra pública.

\begin{abstract}
In the following article, the author analyzes the importance of Building Information Modeling (BIM) and its advantages when combating regulatory rigidity in public works.
\end{abstract}

Palabras claves. - Ingeniería - BIM - Building Information Modelling - Obra pública - modificación.

Keywords. - Engineering - BIM - Building Information Modelling - Public work modification.

* Bachiller en Derecho y especialista en Derecho de la Construcción por la Universidad Peruana de Ciencias Aplicadas (UPC). Especialista en Contrataciones del Estado por la Universidad ESAN. Analista legal de NPG Abogados. Asistente de cátedra en la Universidad del Pacífico en el curso Derecho de la Construcción. Mención especial a mejor orador (2016) y miembro del equipo ganador (2015) de la Competencia Internacional de Arbitraje organizada por la Facultad de Derecho de la Universidad de Buenos Aires y la de Jurisprudencia de la Universidad del Rosario de Bogotá. Miembro de Young International Arbitration Group y de International Council for Commercial Arbitration. 


\section{Introducción}

Desde los primeros ciclos de la carrera de Derecho se nos enseña cual dogma de fe que el Derecho es "el sistema de normas encaminadas a regir la vida de los sujetos y destinadas a la obtención del bien común", es decir, se parte de la premisa que, cual ingenieros sociales, podemos cambiar la realidad a través de las normas jurídicas con el objetivo de conseguir el bienestar general.

No obstante, con el pasar del tiempo, en la práctica profesional, observamos que ello no es del todo cierto, ya que incluso las normas jurídicas pueden generar consecuencias no deseadas, como, por ejemplo, elevar los costos de transacción o potenciar externalidades negativas.

Ese es el caso de la normativa que regula la obra pública, ya que a pesar de las múltiples leyes y reglamentos que la han regido desde 1980 hasta la fecha aún no se consiguen los resultados esperados1. Todo lo contrario, tenemos normas sumamente rígidas e ineficientes que privilegian el cumplimiento de las formalidades y no la ejecución eficiente del proyecto.

Sin embargo, por primera vez en la historia de la obra pública en el Perú apreciamos una modificación, que si bien ha pasado desapercibida en el medio jurídico, representa un giro copernicano respecto la forma cómo se ejecutan e interrelacionan las partes intervinientes en un proyecto. Esta es la incorporación de las herramientas de modelamiento digital a través de los siguientes dispositivos legales:

i. Décimo primera disposición complementaria final del Texto Único Ordenado de la Ley 30225, Ley de Contrataciones del Estado (en adelante, LCE)2: Refiere que "[m]ediante Decreto Supremo se establecen los criterios para la incorporación progresiva de herramientas obligatorias de modelamiento digital de la información para la ejecución de la obra pública que permitan mejorar la calidad y eficiencia de los proyectos desde su diseño, durante su construcción, operación y hasta su mantenimiento."

ii. Numeral 4 del párrafo 8.2 del artículo 8 del Reglamento del Decreto Legislativo 1252, Decreto Legislativo que crea el Sistema Nacional de Programación Multianual y Gestión de Inversiones: Indica que es función de la Dirección General de Programación Multianual de Inversiones del Ministerio de Economía y Finanzas "[e]mitir las metodologías colaborativas

1 De ese modo, hasta la fecha se ha promulgado el Reglamento Único de Licitaciones y Contratos de Obras Públicas (Decreto Supremo 34-80-VC y modificaciones); la Ley de Contrataciones y Adquisiciones (Ley 26850 y modificaciones); Ley de Contrataciones del Estado (Decreto Legislativo 1017 y modificaciones) y la Ley de Contrataciones del Estado (Ley 30225 y modificaciones), así como sus respectivos reglamentos.

2 Vigente desde el 30 de enero de 2019, conforme décima disposición complementaria final del Decreto Legislativo 1444 publicado en el diario oficial El Peruano el 16 de septiembre de 2018. 
de modelamiento digital de la información, para mejorar la transparencia, calidad y eficiencia de las inversiones."

iii. $\quad$ Medida de Política 1.2: Plan BIM del Plan Nacional de Competitividad y Productividad, Decreto Supremo 237-2019-EF: Señala que “[a]nte los persistentes retrasos y sobrecostos en el desarrollo de inversiones en infraestructura, es necesario apuntar a la modernización y digitalización de los sistemas de formulación y evaluación, ejecución y funcionamiento de los proyectos de inversión. Una de las herramientas tecnológicas y digitales a impulsar es la metodología colaborativa de modelamiento digital de la información para la construcción (BIM, por sus siglas en inglés: Building Information Modeling)".

iv. Disposiciones para la Incorporación de BIM en la inversión pública, Decreto Supremo 289-2019-EF: Regula los principios para la adopción y uso del BIM3, y los criterios para su incorporación en los procesos de inversión pública4. Asimismo, aprueba la implementación del Plan BIM Perú, el cual "tiene como propósito reducir los sobrecostos y atrasos en la ejecución de infraestructura pública, hacer más eficiente su operación y mantenimiento, así como propiciar la transparencia en los procesos de inversión pública" . 5

En ese sentido, mediante el presente artículo desarrollaremos el impacto del BIM en la obra pública. Para tal fin, primero, describiremos qué es el BIM y su repercusión en el ciclo de vida del proyecto. Segundo, revisaremos la experiencia comparada en el uso del BIM para corroborar los beneficios que la teoría describe.

\section{II. ¿Qué es el BIM?}

El BIM es una "metodología de trabajo colaborativo que documenta todo el ciclo de vida de la edificación y las infraestructuras, haciendo uso de herramientas informáticas con el fin de generar un repositorio único con toda la información útil para todos los agentes que participan en él y durante todo su ciclo de vida6".

Por su lado, Lui et al. lo definen como: “Una representación digital de las características físicas y funcionales de una instalación. El BIM es un recurso de

3 Según el artículo 3 de las Disposiciones para la Incorporación de BIM en la inversión pública, Decreto Supremo 289-2019-EF, los principios son: (i) eficiencia, (ii) calidad, (iii) colaboración, (iv) transparencia, y (v) coordinación.

4 Según el artículo 4 de las Disposiciones para la Incorporación de BIM en la inversión pública, Decreto Supremo 289-2019-EF, se aplican los siguientes criterios: (i) progresividad, (ii) condiciones de la organización, (iii) integralidad, y (iv) características de la infraestructura.

5 Artículo 5 de las Disposiciones para la Incorporación de BIM en la inversión pública, Decreto Supremo 289-2019-EF

6 GOSALVES, Jordi, et. al. BIM en 8 puntos. Todo lo que necesitas conocer sobre BIM. España: Es.BIM, 2016, p. 2. 
conocimiento compartido para obtener información sobre una instalación que constituye una base confiable para tomar decisiones durante su ciclo de vida7".

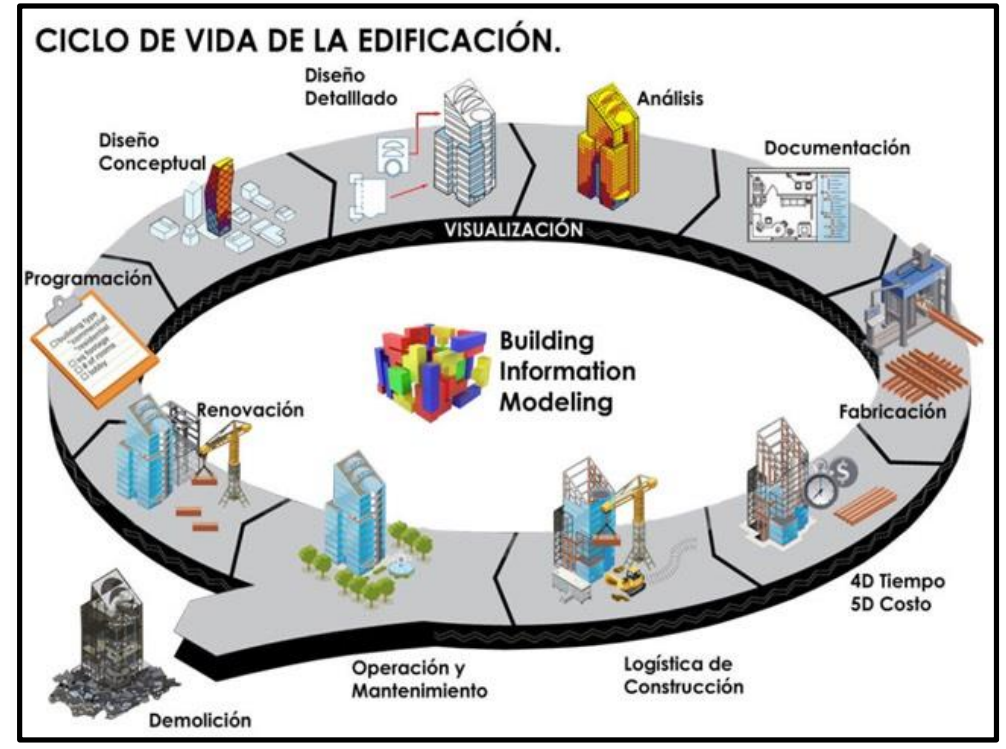

Figura 1: Ciclo de vida de un proyecto.

Fuente: CiberSal, 2018

Por tal razón, el BIM

"es ese amigo que te aconseja y ordena tus proyectos porque tiene una extensa experiencia internacional, porque tiene esa información que necesitas al instante, ya que posee más de 35 años en el mercado global y uno de sus pilares es compartir, difundir, colaborar con proyectos desde el inicio del diseño y, sobre todo, crear una cultura de prevencións".

A partir de las definiciones y descripciones precedentes acerca de lo que es el BIM extrapolamos las siguientes premisas:

i. El BIM es una representación física y funcional de la obra.

ii. El BIM es una metodología de trabajo colaborativo.

iii. El uso del BIM contribuye a optimizar los resultados esperados durante todo el ciclo de vida del proyecto.

7 LUI, Fangxiao, JALLOW, Abdou, ANUMBA, Chimay y WU, Dinghao. (2012) “Building knowledge modeling: integrating knowledge in BIM", Beijing, 9-12 de octubre de 2013, p.1. Consulta realizada el 22 de enero de 2020. Disponible en web: https://pdfs.semanticscholar.org/c020/2c81ca356608148dfaacd27f187fc2202ec8.pdf?_ga=2.763 49525.1548575591.1579748407-309372318.1579748407

Traducción libre: "a digital representation of physical and functional characteristics of a facility. A BIM is a shared knowledge resource for information about a facility forming a reliable basis for decisions during its lifecycle".

8 CÉSPEDES, Jesús, y CORREA, Paul. (2018) "Ventajas competitivas con BIM / VDC". En Revista al servicio del desarrollo y promoción de la construcción Constructivo, vol. 20, núm. 131, octubre noviembre 2018, p. 132. 
A continuación, desarrollaremos cada una de estas premisas.

\section{i. El BIM es una representación física y funcional de la obra}

La representación física y funcional de la obra requiere la integración de una serie de programas informáticos a través de los cuales se esquematiza el proyecto hasta en ocho dimensiones, siendo estas las siguientes:

\section{a) BIM 3D (arquitectura + estructura + instalaciones)}

Representa la información del diseño arquitectónico y cada una de las disciplinas que integran el proyecto.

Según, Fernández et. al.:

“Representa la integración de las diferentes especialidades en torno a un modelo tecnológico (...), el cual puede ser creado de manera colaborativa entre los diferentes profesionales. Esta interacción virtual consolida la gestión del diseño, pues permite visualizar las modificaciones que se deberían ejecutar al producirse cambios en el diseño y brindar soluciones a las incompatibilidades desde las fases tempranas del proyecto ${ }^{\prime \prime}$.

\section{b) BIM 4D (BIM 3D + programación)}

La incorporación de la programación permite superar la visión estática del proyecto reconociendo su naturaleza dinámica. Para ello se vincula la estructura en 3D con programas como Microsoft Project o Primavera P6 a través de los cuales se controla el tiempo.

Por ejemplo, si la entidad determina que es necesario realizar las excavaciones para la construcción de los sótanos con un metro de profundidad adicional al planificado, desde el preciso instante en el que se modifican los planos en el modelo también se modifica el plazo de las actividades afectadas.

Con el uso del BIM 4D la discusión sobre la cantidad de días de la ampliación de plazo y la afectación de la ruta crítica desaparecerá, ya que solo bastará revisar el Primavera P6 o el programa elegido por las partes para la programación. Por lo que la discusión estará limitada a verificar si el retraso justificante de la ampliación es imputable a la entidad o a un evento de caso fortuito o fuerza mayor10.

BIM 5D (BIM 4D + costos)

9 FERNÁNDEZ, Leandro, RÍOS, Renzo, MARREROS, John. “Más allá de la tecnología: BIM como una nueva filosofía". En Revista Civilízate, núm. 8, 2016, p. 47.

10 Por lo que será necesario modificar el artículo 198 (Procedimiento de ampliación de plazo) del Reglamento con el objetivo de adecuar el procedimiento al uso del BIM, en vista que, ya no se requerirá el cuaderno de obra. Asimismo, tendrá que reducirse los plazos de la entidad para resolver la procedencia de la ampliación de plazo ya que con el BIM será más simple determinar la afectación de la ruta crítica. 
Uno de los elementos más importantes en cualquier proyecto es el costo, por lo que se requiere un control minucioso con el objetivo que la obra se ejecute dentro de lo presupuestado. Para ello se suele usar los programas Presto, Arquímedes, entre otros.

El BIM 5D permite elaborar el presupuesto del proyecto a partir de la ingeniería de detalle (etapa de diseño) y controlar los costos incurridos durante el transcurso de la ejecución de la obra (etapa de construcción).

Por ejemplo, el BIM 5D permitirá cuantificar de manera rápida y precisa los adicionales de la obra generados por las órdenes de cambio de la entidad, en vista que desde el momento en que se incorpora la variación en el modelo, este también arrojará la información acerca del costo de la ejecución de dicha variación.

Ahora bien, el BIM 5D no solo se limita a cuantificar aspectos relativos a la ejecución,

“En la fase operativa o de explotación también permite la toma de decisiones sobre el control de costes. En primer lugar, porque anticipa a esa fase calculando los costes de funcionamiento y mantenimiento de componentes del proyecto, lo que permite valorar su adecuación en función de la rentabilidad y durabilidad de los mismos. También permite analizar, calcular y decidir cambios, modificaciones o reformas que contribuyan a un mejor rendimiento y eficacia del edificio en funcionamiento11".

\section{d) BIM 6D (BIM 5D + sustentabilidad)}

En este nivel se incorporan criterios de "Green building" con el objetivo que la infraestructura sea sostenible y amigable con el entorno. El BIM 6D

“permite testar y simular su comportamiento pasivo desde el principio, permitiendo la toma de decisiones en cuanto a diseño en fases tempranas que luego provocarán la necesidad de rehacer el trabajo ya hecho. $\mathrm{Y}$ en cuanto a su comportamiento activo, una vez integradas las instalaciones de confort térmico, se podrán realizar tantas simulaciones de rendimiento como se deseen alternando los equipos instalados. La combinación de ambos nos permitirá evaluar el modelo BIM creando variaciones e iteraciones en la envolvente, los materiales utilizados, el tipo de combustible para enfriar/ calentar los espacios del objeto de la construcción, etc. Teniendo en cuenta, además, su emplazamiento geográfico, su posición y orientación, el nivel de soleamiento en las distintas estaciones del año, las temperaturas de la zona, etc.12".

e) BIM 7D (BIM 6D + mantenimiento)

11 OLIVER, Inmaculada. (2015) (Tesis) “Integración de la metodología BIM en la programación curricular de los estudios de Grado en Arquitectura Técnico/ Ingeniería de Edificación. Diseño de una propuesta." Valencia: Universidad Politécnica de Valencia. p. 96.

12 Ídem. 
Mediante esta dimensión se gestiona el ciclo de vida útil de los activos del proyecto. Por ejemplo, luego de ejecutada la obra en el mismo modelo se puede incorporar alertas a través de las cuales asignamos fechas específicas que nos recuerden la realización del mantenimiento. De ese modo, se "hace click" en una grúa y se anota que cada dos meses se debe cambiar el aceite y los filtros. Por lo que, cuando se cumplan dichos plazos el modelo emitirá las alertas para que el operador realice el mantenimiento.

Ahora bien, este tipo de acciones no solo permite prolongar el tiempo de vida del activo sino además planificar futuros desembolsos mejorando el control de costos del proyecto.

La implementación del BIM 7D permitirá a las entidades públicas tener una visión a largo plazo de la obra. Un caso en donde falló este aspecto ocurrió en Italia (Génova) el 2018 cuando el puente A-10 (conocido como Morandi) se derrumbó (siendo la falta de mantenimiento una de las causas principales) provocando al menos 39 muertos y más de una decena de heridosi3.

En tal sentido, el BIM no solo permitirá un adecuado mantenimiento de las obras públicas sino además la prevención de accidentes (daños patrimoniales y extrapatrimoniales) como el descrito en el párrafo precedente.

\section{f) BIM 8D (BIM 7D + seguridad y salud ocupacional)}

Esta última dimensión nace como respuesta al alto nivel de accidentalidad de la industria de la construcción. Por tal razón, se ha visto conveniente adoptar medidas preventivas desde la etapa de diseño a través del BIM 8D.

Según Kamardeen el BIM 8D incluye fundamentalmente tres tareas: “(1) elaboración de perfiles de peligro del modelo BIM, (2) presentación de sugerencias de diseño seguro para revisar los elementos perfilados de alto riesgo, y (3) propuesta de controles de riesgo in situ para los peligros incontrolables mediante revisiones del diseño1".

Como se aprecia, a través de las dimensiones del BIM se tiene un registro de información del pasado, presente y futuro (ciclo de vida) de la obra desde todas sus aristas, lo que permite un mejor control del proyecto 15.

13 FOUNTAIN, Henry. (25 de agosto de 2018) Diseño y mantenimiento del puente en Génova, Italia, están bajo sospecha. El Nuevo Herald. (Disponible en: https:// www.elnuevoherald.com/opinion-es/trasfondo/article217070475.html, consulta realizada el 22 de enero de 2020).

14 KAMARDEEN, Imriyas. "8D BIM Modelling tool for accident prevention though design. Egbu, C. (Ed) Procs 26th Annual ARCOM Conference", Leeds, UK, 2010, Association of Researchers in Construction Management, p. 285.

Traducción libre de: "(1) hazard profiling of BIM model elements, (2) providing safe design suggestions for revising high hazard profiled elements, and (3) proposing on-site risk controls for hazards that are uncontrollable through design revisions".

15 Tal nivel y profundidad en la información del proyecto coadyuvará a prevenir y resolver más rápida y eficientemente los conflictos que pudiesen surgir durante la ejecución de la obra. Por 


\section{ii. El BIM es una metodología de trabajo colaborativo}

Esta característica desmitifica la errada visión que equipara al BIM con un software de diseño ya que es mucho más que eso, pues implica un cambio en la forma como se ejecutan las obras a partir de su conocimiento en tiempo real por parte de todos los agentes críticos del proyecto.

En esa misma línea, Madeira et al (2016) afirma que:

“BIM no es simplemente una nueva forma de diseño o algo que afecta solo al diseño y construcción de un edificio, sino un proceso centrado en el desarrollo, uso y transferencia de un modelo de información digital de un proyecto de construcción para mejorar el diseño, la construcción y las operaciones de un proyecto o cartera de instalaciones16".

En tal sentido, si antes se acostumbraba a una ejecución descoordinada del proyecto, con el BIM se propende a un trabajo conjunto lo que se materializa, por ejemplo, en que los agentes críticos trabajen en un área común en donde se intercambia información de manera constante y se reporta a través de reuniones semanales el avance de la obra. Asimismo, se analiza las contingencias presentes y futuras con el objetivo de buscar las mejores soluciones.

En esa misma línea, el numeral 3 del artículo 3 de las Disposiciones para la Incorporación de BIM en la inversión pública, Decreto Supremo 289-2019-EF, señalan que: "La adopción y uso de BIM debe garantizar la máxima participación, comunicación e intercambio de información entre los diversos involucrados en el desarrollo de una infraestructura pública, en cada una de las diferentes etapas y fases del ciclo de inversión."

Sin embargo, el nivel de colaboración no será el mismo en todos los proyectos, ya que dependerá del Project Delivery System (en adelante, PDS) elegido por el propietario y materializado en el contrato de construcción.

¿Qué es el PDS?, es el método mediante el cual se define la estructura de las relaciones entre las partes intervinientes de un proyecto de construcción, sus

ejemplo, el trabajo de la Junta de Resolución de Disputas se verá optimizado ya que además de contar con información contemporánea del proyecto también esta tendrá un alto nivel de confiabilidad. Ello además se ve potenciado con la participación de técnicos (ingenieros, sobre todo) como miembros de la Junta de Resolución de Disputas, los cuales están capacitados para interpretar la data del BIM.

16 MADEIRA, Victor, FRAME, Shona, HENEGHAN, Jarleth, JOHANSEN, Christian, MOORE, Joseph, y VOGEL, Sharon. "Legal aspects of Building Information Modelling: a world view (Part I)”. En Construction Law International, vol. 11, núm. 4, diciembre de 2016, p. 10.

Traducción libre: "BIM is not simply a new way to design or something which affects only the design and construction of a building, but a process focused on the development, use and transfer of a digital information model of a building project to improve the design, construction and operations of a project or portfolio of facilities". 
funciones y la secuencia general de actividades17. Dicha definición estará a cargo exclusivamente de la entidad en base a sus requerimientos.

De esa manera, la decisión sobre si las labores de diseño y construcción recaerán en un solo contratista mediante un contrato o en dos contratistas con contratos independientes estará en manos de la entidad.

Los PDS más utilizados por la industria son los siguientes:

a) Tradicional

b) Diseño y construcción

c) Gerencia

d) Integrado

A continuación, verificaremos cómo se aplica el BIM en cada PDS.

\section{a) Sistema tradicional (Design - Bid - Build)}

En este PDS el contratista únicamente está obligado a ejecutar la obra de conformidad con la ingeniería de detalle entregada por la entidad, lo cual implica que cualquier indefinición y/o error será asumido por esta última. De ese modo, la entidad está obligada a subsanar o entregar la ingeniería faltante.

Este es el supuesto regulado en el artículo 32.7 de la LCE al indicar:

“La responsabilidad por la adecuada formulación del Expediente Técnico o Estudios Definitivos corresponde al proyectista y a la supervisión, de acuerdo con el alcance de los respectivos contratos, y la aprobación a la Entidad. De igual modo, la entrega completa de la información que es puesta a disposición de los postores corresponde a la Entidad."

Somos de la opinión que el BIM es beneficioso en un contrato de construcción bajo el sistema tradicional puesto que optimiza los derechos y deberes de las partes. Como es el caso del deber del contratista de avisar inmediatamente sobre los errores y/o indefiniciones en la ingeniería entregada por la entidad 18. Si bien este deber no se encuentra regulado expresamente en el Código Civil puede interpretarse a partir de los siguientes artículos:

1. Numeral 2 del artículo 1774 del Código Civil: "El contratista está obligado: (...) 2.- A dar inmediato aviso a comitente de los defectos del suelo o de la

17 MOORE, Dale. (2000) "Selecting the best Project delivery system", Project Management Institute Annual Seminars \& Symposium, Houston, 7 de septiembre de 2000.

18 El artículo 40 (Responsabilidad del contratista) del Reglamento hace referencia al Código Civil al indicar que: “(...) se debe cumplir lo dispuesto en los numerales 2) y 3) del artículo 1774 del Código Civil. 
mala calidad de los materiales proporcionados por éste, si se descubren antes o en el curso de la obra y pueden comprometer su ejecución regular."

2. Artículo 1362 del Código Civil: "Los contratos deben (...) ejecutarse según las reglas de la buena fe $(. . .)^{\prime \prime}$

En tal sentido, si el contratista verifica (a través del BIM) que hay algún error en la ingeniería durante la ejecución de la obra, este tiene el deber de informarlo inmediatamente a la entidad, para que esta provea la ingeniería faltante o corregida.

En esa misma línea, Arias- Schereiber y Cárdenas, comentando el artículo 1774, señalan que:

"Se han hecho críticas al artículo 1774, por cuanto no establece que el contratista debe dar aviso al comitente sobre los defectos de la información técnica que le ha sido proporcionado para la ejecución de la obra. De acuerdo con nuestro criterio la objeción es válida, aunque la solución podría estar en la aplicación por analogía del artículo bajo comentario19".

Ahora bien, ¿cuál es el estándar de conducta esperado para el cumplimiento del “deber de avisar"? El regulado en el artículo 1314 del Código Civil: “Quién actúa con la diligencia ordinaria requerida, no es imputable por la inejecución de la obligación o por su cumplimiento parcial, tardío o defectuoso."

En otras palabras, como afirman Castillo y Rivas,

“el comportamiento del obligado se confrontará con la lex artis, es decir, con el conocimiento y ciencia que sean exigibles al profesional. Se añade que en ello gravitan el estado y desarrollo de los conocimientos con el propósito de responsabilizar al profesional que ignore los avances de la ciencia que se encuentren a su disposiciónzo".

En tal sentido, el contratista incurrirá en responsabilidad si: "no cumple con dar aviso" o "no avisa inmediatamente" y si a raíz de dicho incumplimiento se generan daños a la entidad, de conformidad con el artículo 1321 del Código Civil: "Queda sujeto a indemnización de daños y perjuicios quien no ejecuta sus obligaciones por dolo, culpa inexcusable o culpa leve"

El Navisworks es uno de los softwares integrados al BIM 3D que permite identificar, por ejemplo, las interferencias entre los planos de estructuras y los planos de instalaciones sanitarias. De ese modo, si un tubo de agua intercepta una columna el contratista tendrá que dar aviso inmediatamente para que la entidad corrija dicho error.

19 ARIAS-SCHREIBER, Max y CÁRDENAS, Carlos. Exégesis del Código Civil peruano de 1984. Tomo III. Lima: San Jerónimo, 1989, p. 106.

20 CASTILLO, Mario y RIVAS, Gino. (2014) “La diligencia y la inejecución de las obligaciones". En Ius Et Veritas, núm. 48, julio 2014, pp. 130- 141. 
Imaginemos cuánto tiempo demorarían las partes resolviendo estos problemas en el contexto de la obra pública sin la tecnología. Con Navisworks desde el momento que el contratista identifica la interferencia, la entidad también toma conocimiento de ella. En ese escenario, las partes evitarán discusiones sobre la existencia del error en la ingeniería y se reducirá el tiempo en su absolución.

Por otro lado, la entidad con el apoyo del BIM podrá reducir el alcance de la obra anticipadamente para ajustar la retribución pactada cuando el contrato sea por ajuste alzado, conforme la última parte del artículo 1776 del Código Civil: “El comitente, a su vez tiene derecho al ajuste compensatorio en caso de que dichas variaciones signifiquen menor trabajo o disminución en el costo de la obra." Ello le permitirá a la entidad adecuar el proyecto a las nuevas necesidades que pudiesen surgir durante su ejecución.

Por tales razones discrepamos con Gray y Bravo cuando afirman que: “Como los contratistas no asumen el riesgo del diseño bajo este sistema de entrega de proyectos, su posición no mejora ni se beneficia del uso del BIM por parte del propietario21". Debido a que como hemos demostrado el BIM genera beneficios en el ejercicio de los derechos y deberes de las partes.

\section{b) Sistema Diseño y Construcción (Design - Build)}

En este PDS la entidad le entrega al contratista una ingeniería preliminar (conceptual, básica o básica extendida) para que desarrolle la ingeniería de detalle y construya la obra con ella. En este caso, cualquier error y/o indefinición en la ingeniería es asumida por el contratista.

En obra pública, este sistema se encuentra regulado en las modalidades de contratación llave en mano y concurso oferta, conforme el artículo 36 del Reglamento:

“a) Llave en mano: (...) Tratándose de obras, el postor oferta en conjunto la construcción, equipamiento y montaje hasta la puesta en servicio y, de ser el caso, la elaboración del expediente técnico y/o la operación asistida de la obra; b) Concurso oferta: Cuando el postor oferta, la elaboración de expediente técnico y ejecución de la obra."

El BIM permitirá, al igual que en el sistema tradicional, optimizar las labores de las partes. Al contratista, le brindará las herramientas tecnológicas para preparar una ingeniería de detalle con la menor cantidad de errores y/o indefiniciones.

El BIM 4D le posibilitará al contratista compatibilizar mejor las interfaces entre las labores de diseño y construcción, sobre todo cuando se ejecutan mediante fast-

21 GRAY, Jaime y BRAVO, Jonnathan. "Liabilities for design defects in a collaborative integrated digital age". En Construction Law International, vol. 8, núm. 4, diciembre 2013, pp. 19- 24.

Traducción libre: "As the risk of the design is not taken by contractors under this project delivery system, their position is not improved nor do they benefit from the use of BIM by owner" 
track 22. Por ejemplo, si se contrata a un subcontratista para que prepare el diseño y construya la obra gruesa de una edificación, y a otro subcontratista para los acabos, a través del BIM 4D se podrá controlar las interfaces de labores con el objetivo de evitar cualquier retraso, más aún si el primer subcontratista superpone las actividades de diseño y construcción.

Por el lado de la entidad, el uso del BIM le permitirá tener un mayor control respecto el cumplimiento de sus requerimientos técnicos (ingeniería preliminar).

\section{c) Sistema de Gerencia}

En este sistema la entidad contrata una empresa que actúa como facilitador del proyecto, con la finalidad que lo gerencie, ya sea como un asesor técnico/legal (agency manager) o asumiendo la responsabilidad por la ejecución, incluso desde la etapa de diseño (at risk manager).

La diferencia radica en que al gerente al riesgo se le contrata para entregar un proyecto, en otras palabras, asume frente a la entidad el riesgo del diseño y de la construcción. En cambio, el agente, solo se limita a asesorar a la entidad sin asumir ningún riesgo, en vista que será la entidad, quien finalmente decida qué proyectista y contratista es el idóneo para su proyecto. En tal sentido, el gerente al riesgo actúa como un verdadero contratista, conforme el artículo 1771 del Código Civil 23, y el agente como un locador de servicios, de acuerdo con el artículo 1764 del Código Civil 24.

En ambos casos, el BIM les permitirá un mejor control del proyecto. Asimismo, podrán adoptar decisiones más informadas en tanto cuentan con data contemporánea de la obra. De ese modo, el agente brindará mejores recomendaciones a la entidad a fin de que esta tome decisiones informadas. En cambio, el gerente al riesgo será quien las elabore e implemente directamente.

Un ejemplo exitoso, ajeno a la LCE y su Reglamento25, se registró en la ejecución de las obras para los Juegos Panamericanos Lima 2019, en donde se utilizó como marco legal y contractual los New Engineering Contract (NEC), opción F (gerencia al riesgo) y los Project Management Office (PMO).

\section{d) Sistema Integrado}

A diferencia de los anteriores sistemas en donde el contrato de obra es celebrado por la entidad y el contratista, el contrato bajo el sistema integrado se caracteriza

22 Es un sistema de gestión en el que la preparación de la ingeniería se superpone con la ejecución de la obra para reducir el tiempo.

23 Artículo 1771 del Código Civil: "Por el contrato de obra el contratista se obliga a hacer una obra determinada y el comitente a pagarle una retribución".

24 Artículo 1764 del Código Civil: "Por la locación de servicios el locador se obliga, sin estar subordinado al comitente, a prestarle sus servicios por cierto tiempo o para un trabajo determinado, a cambio de una retribución."

25 Ello es así porque la LCE ni su Reglamento regulan el PDS del gerente al riesgo. Para los Juegos Panamericanos Lima 2019 se recurrió a un Acuerdo de Estado a Estado con el Gobierno Británico. 
por ser multipartito, es decir, lo celebran todos los agentes críticos del proyecto (contratista, comitente, proyectista, gerente agente o al riesgo, entre otros) con la finalidad de optimizar el nivel de colaboración a partir de una asignación conjunta de riesgos positivos y negativos.

En otras palabras, los ahorros se comparten y las pérdidas de distribuyen entre todas las partes del acuerdo multipartito. A menos que, "exista una disposición explícita (no implícita) hecha por las partes que transfieren el riesgo a una de ellas exclusivamente26".

En ese contexto, el sistema integrado tiene características particulares que lo distinguen de otros PDS, siendo, sin ser los únicos, los siguientes:

1. Los agentes intervinientes del proyecto (principales) se encuentran vinculados desde etapas iniciales (conceptualización) y tienen participación en la toma de decisiones, las cuales se asumen por consenso o en su defecto, por mayorías.

2. Se le otorga a la etapa de diseño una real importancia. Por lo que, tanto el propietario como el proyectista (incluso el contratista) intervienen intensamente en la elaboración de una ingeniería adecuada y con la menor cantidad de errores.

3. Las relaciones contractuales tienen una estructura distinta frente a otros PDS, en vista de que ya no se trata de contratos independientes (de ingeniería, construcción o gerencia), sino de un contrato que integra a los principales agentes intervinientes del proyecto.

De esa manera, veremos, por ejemplo, que un contratista tendrá incentivos de brindar sus recomendaciones en la etapa de diseño al proyectista porque sabe que, si la obra se ejecuta dentro del "costo meta" será acreedor de un incentivo económico. Del mismo modo, el proyectista tendrá una labor proactiva corrigiendo la ingeniería antes que el contratista identifique el error durante la ejecución de la obra evitando así cualquier retraso.

$\mathrm{Al}$ igual que la gerencia al riesgo, el sistema integrado tampoco se encuentra regulado en la LCE ni su Reglamento, por lo que se tendrá que recurrir a otra legislación como el caso de los Acuerdos de Estado a Estado. Por ejemplo, podrían usar como marco contractual el ConsensusDocs 300 con el Building Information Modeling (BIM) Addendum.

Ahora bien, ¿cuál es el PDS ideal para la implementación del BIM? Madeira et. al. sostienen que "hay consenso en que, si bien BIM puede aportar beneficios a los contratos diseño- licitación- construcción tradicionales dirigidos por el empleador

26 GRAY, Jaime y BRAVO, Jonnathan. "Liabilities for design defects in a collaborative integrated digital age". En Construction Law International, vol. 8, núm. 4, diciembre 2013, pp. 19- 24.

Traducción libre: "there is an explicit (not implicit) provision made by the parties transferring the risk to one of them exclusively" 
(en la medida en que BIM se regule correctamente), ofrece mayores beneficios cuando el contratista participa tempranamente (...) o cuando existen contratos de colaboración $27 "$

De esa manera, el papel del constructor en las etapas tempranas del proyecto es fundamental ya que brindará recomendaciones para que la obra tenga un alto nivel de constructividad 28 .

Asimismo, como afirma Quiroz:

“El diseño básico del proyecto, aun siendo desarrollado desde modelos BIM, necesita de una validación constructiva que solo podrá darse con la inclusión del constructor, los subcontratistas y los proveedores. Por ello, el diseño de los proyectos desarrollados en BIM deben contemplar el proceso de conversión de los modelos de diseño inicial a modelos de producción $29 "$ ".

\section{iii. El uso de BIM contribuye a optimizar los resultados durante todo el ciclo de vida del proyecto}

La El BIM tiene una importante incidencia económica en el BAM (Building Assembly Model) y en el BOOM (Building Operation Optimization Model), ya que como afirma MacLeamy, por cada \$ 1 dólar que se invierte en la etapa de diseño se invierte $\$ 20$ en construcción y \$ 60 o más a lo largo de la vida útil del proyecto por mantenimiento30, conforme se aprecia en la siguiente figura:

27 MADEIRA, Victor, FRAME, Shona, HENEGHAN, Jarleth, JOHANSEN, Christian, MOORE, Joseph, y VOGEL, Sharon. "Legal aspects of Building Information Modelling: a world view (Part II)”. En Construction Law International, vol. 12, núm. 1, marzo de 2017, pp. 19-20.

Traducción libre: "there is a consensus that while BIM can bring benefits under employer led traditional design-bid-build procurement contracts (in so far as BIM is specified correctly), it offers greater benefits through early contractor engagement (...) or where there are collaborative contracts."

28 LOYOLA, Mauricio y GOLDSACK, Luis. Constructividad y Arquitectura. Santiago: Universidad de Chile, 2010, p. 12.

$\mathrm{Al}$ respecto, Loyola y Goldsack precisan que la: "Constructividad describe la manera en que "un diseño" facilita su posterior construcción; no la manera en que "un proyecto", "un tipo de administración" o incluso "un equipo profesional" facilita su construcción. (...) Un diseño tiene un alto grado de constructividad si "considera atentamente el modo en que se ha de construir el edificio y los condicionantes prácticos que actúan en este proceso".

29 QUIROZ, Felipe. (2018) "Propuesta para la aceleración e industrialización de la construcción con modelos BIM de producción". En Revista al servicio del desarrollo y promoción de la construcción Constructivo, vol. 20, núm. 131, octubre-nomviembre 2018, pp.140- 142.

30 MILLASAKY, C. (2018) (Tesis) Cuantificación de los beneficios económico de subcontratar servicios BIM (Building Information Modeling) en la etapa de diseño para proyectos de edificaciones en Lima Metropolitana. Lima: Pontificia Universidad Católica del Perú. 


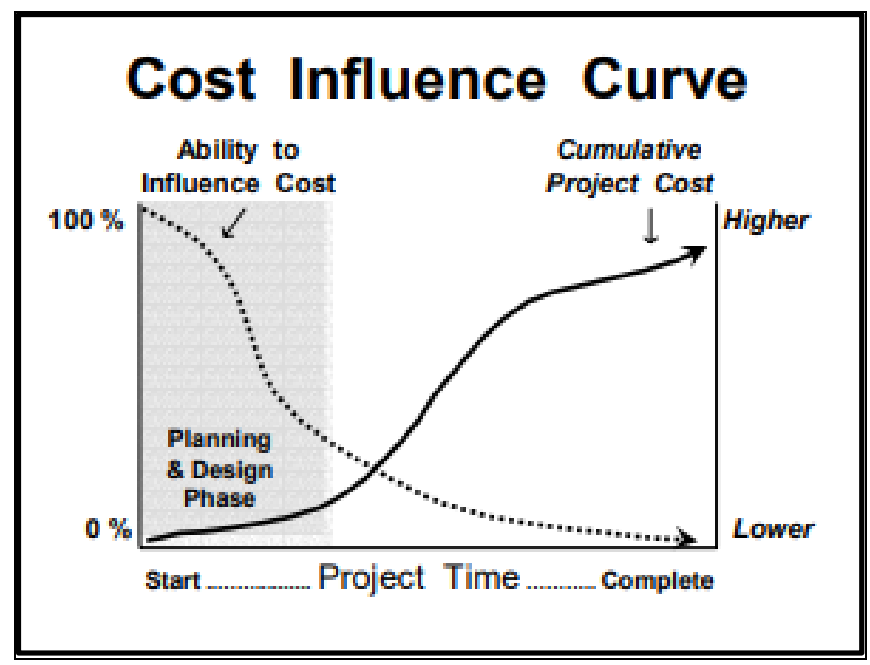

Figura 2: Curva de la influencia de costos.

Fuente: Rocque, Bernice - Enabling effective Project sponsorship

En ese mismo sentido, Millasaky señala que

"[1]o que se busca es invertir más tiempo y esfuerzo en la etapa inicial del proyecto para conseguir grandes beneficios en las próximas etapas BAM y BOOM. Esto no necesita mucha inversión al inicio, ya que los costos en la etapa de diseño son mucho menores que en las próximas etapas. Por lo tanto, los cambios en esta etapa no representan un gran impacto en el costo total del proyecto31".

Por ejemplo, si se identifica un error, dependiendo de la etapa del proyecto en la que nos encontremos, el costo de remediación será diferente:

\begin{tabular}{|l|ll|}
\multicolumn{1}{c|}{$\begin{array}{c}\text { Etapa del } \\
\text { proyecto }\end{array}$} & \multicolumn{1}{c|}{ Algunos costos potenciales } \\
\hline Diseño & - & \multicolumn{1}{c|}{ Corrige o prepara otro diseño. } \\
\hline & - & Corrige o prepara otro diseño. \\
Construcción & - & $\begin{array}{l}\text { Elimina la parte de la obra con vicios. } \\
\text { - }\end{array}$ \\
& & $\begin{array}{l}\text { Se vuelve a construir a partir del diseño nuevo o } \\
\text { corregido. }\end{array}$ \\
\hline Operación & - $\begin{array}{l}\text { Corrige o prepara otro diseño } \\
\text { - }\end{array}$ & $\begin{array}{l}\text { Elimina la parte de la obra con vicios } \\
\text { corregido a construir a partir del diseño nuevo o }\end{array}$ \\
\hline
\end{tabular}

$31 \quad$ Ibid., p. 20. 


\begin{tabular}{|l|l|}
\hline - & $\begin{array}{l}\text { La obra no cumple su finalidad si la parte viciada } \\
\text { es necesaria para el funcionamiento de las demás } \\
\text { partes. }\end{array}$ \\
- & $\begin{array}{l}\text { Si se decide no eliminar la parte viciada puede } \\
\text { aumentar el mantenimiento de dicha parte para } \\
\text { evitar que la obra no cumpla con su finalidad. }\end{array}$ \\
\hline
\end{tabular}

Tabla 1: Costos incurridos por la detección de un error en las etapas del proyecto

De ese modo, "[s]e debe asegurar que el BIM genere ahorros en el uso de los fondos públicos a lo largo del ciclo de inversión, en términos de reducción de sobrecostos y atrasos en la ejecución de la infraestructura pública, así como en un uso racional de recursos destinados a operación y mantenimiento32".

\section{El BIM en la experiencia comparada en materia de obra pública}

El BIM no es ajeno a la obra pública en la experiencia comparada, en tanto ya es utilizado con mucho éxito en la Unión Europea, EE. UU. y en algunos países de Latinoamérica, como es el caso de Chile, debido a sus importantes y concretos beneficios.

El EU BIM Taskgroup refiere que la propuesta de valor del BIM se traduce

"en beneficios económicos, como un aumento de la rentabilidad de los fondos públicos durante la fase de ejecución o la mejora de la calidad de los bienes y servicios públicos durante la utilización del activo construido. Para un responsable político al que le preocupe el rendimiento del sector de la construcción, estos beneficios económicos se pueden agregar a nivel nacional para respaldar unos niveles de productividad mayores (medidos como PIB) y un mayor potencial de crecimiento (medido a través de las exportaciones) $33^{\prime \prime}$.

En otras palabras, el costo de implementación del BIM (adecuación informática y capacitación al personal vinculado a la ejecución de la obra pública) es menor al beneficio micro y macroeconómico derivado de su utilización. Es decir, el BIM es una inversión rentable a corto, mediano y largo plazo.

Asimismo, el BIM genera beneficios medioambientales y sociales para las entidades públicas en general. Respecto los primeros, el uso del BIM reduce el "volumen de residuos que termina en los vertederos gracias a una mayor precisión en los pedidos de materiales, o una simulación optimizada de estudios energéticos que se traduce en una menor demanda de energía por parte del entorno construido34".

32 Numeral 1 del artículo 3 de las Disposiciones para la Incorporación de BIM en la inversión pública, Decreto Supremo 289-2019-EF.

33 EU BIM TASKGROUP (2016) Manual para la introducción de la metodología BIM por parte del sector público europeo. Co-funded by the European Union. p. 18.

34 Ídem. 
Por ejemplo, a través del BIM 6D se pueden ejecutar las obras considerando su sostenibilidad. En términos de LEED Green Certification System ello significa que:

i. Se controla la cantidad de agua requerida para la construcción. Se adopta las medidas necesarias para su uso eficiente.

ii. La energía utilizada en el proyecto tenga el menor impacto atmosférico.

iii. Los materiales y recursos utilizados para la construcción no afecten el medio ambiente.

iv. El diseño de los interiores de la estructura tenga como objetivo el uso de la menor cantidad de energía en base al entorno climático (cálidos o fríos) en el que se ejecute la obra.

Por otro lado, también se pueden obtener beneficios sociales

"mediante una utilización eficaz de BIM en la planificación y consultas públicas con el fin de obtener apoyo para las infraestructuras públicas nuevas o renovadas (por ejemplo, ubicación de autopistas, sistemas de contención de agua o reforma de edificios públicos). Esta participación pública puede respaldar una infraestructura pública correctamente diseñada y que responda a las necesidades de la comunidad local. Esto puede traer consigo mejores resultados sociales, como una mejora en la planificación de los recursos, un mayor uso de las instalaciones públicas o la cartografía y protección de elementos del patrimonio histórico arquitectónico35".

A nivel mundial, el líder en el desarrollo del BIM en la industria de la construcción es EE. UU., quien desde el año 2003 lo implementa tanto en el ámbito público como privado. Además, cuenta con guías metodológicas y protocolos que permiten la interoperatividad de todos los agentes intervinientes del proyecto y los órganos reguladores como el General Services Administration (GSA) del gobierno federal 36 .

En Europa, la implementación del BIM en la obra pública comenzó en Reino Unido el 2011, en donde se obtuvieron resultados sumamente favorables, ya que entre el periodo de 2011 y 2014 hubo una reducción en el costo de los proyectos entre el $12 \%$ y el $20 \%$ lo que se tradujo en ahorros del alrededor de 2,300 millones de dólares37.

Posteriormente, como respuesta frente a los resultados favorables del BIM, la Unión Europea a través de la Directiva 2014/24/UE del Parlamento Europeo y del Consejo estableció en el numeral 4 del artículo 22 que: "Para los contratos públicos de obra y concursos de proyectos los Estados miembros podrán exigir el uso de herramientas electrónicas específicas, como herramientas de diseño electrónico de

35 Ídem.

36 PAREDES, Gustavo. (2019) “El BIM se pone el overall y encima la toga: La tecnología y el cambio en la gestión de las disputas en construcción”. En Revista de Derecho Sociedad Jurídica, núm. 6, 2019, pp. 137- 156.

37 Ídem. 
edificios o herramientas similares" De esa forma, el uso del BIM en materia de obra pública se convirtió en una realidad en la Unión Europea.

Entre los países que ya implementaron en sus legislaciones lo señalado por la Directiva 2014/24/UE se encuentra España, quien en el numeral 6 de la Disposición Adicional Decimoquinta de la Ley 9/2017, de Contratos del Sector Público, refiere: "Para contratos públicos de obras, de concesión de obras, de servicios y concursos de proyectos, y en contratos mixtos que combinen elementos de los mismos, los órganos de contratación podrán exigir el uso de herramientas electrónicas específicas, tales como herramientas de modelado digital de la información de la construcción (BIM) o herramientas similares."

Finalmente, en Latinoamérica, desde el año 2016 en Chile se viene ejecutando el Plan BIM en el marco del Programa Estratégico “Construye 2025”, a través del cual se "busca impulsar y dar valor a la industria con la introducción de metodologías y tecnologías para el desarrollo y operación de proyectos de edificación e infraestructura. En un principio, se aplicará en el sector público de forma obligatoria a $202038^{\prime \prime}$.

\section{Conclusiones}

La El BIM ha llegado para revolucionar la forma como se ejecuta la obra pública y cómo se interrelacionan los sujetos intervinientes en el proyecto, por lo que es imprescindible que los operadores jurídicos estemos preparados para gestionarlo adecuadamente.

Para ello debemos comenzar comprendiendo que el BIM no solo es un software, sino además es un método de trabajo colaborativo que contribuye a optimizar los resultados esperados durante todo el ciclo de vida del proyecto.

El BIM en la experiencia comparada ha demostrado ser exitoso, generando grandes ahorros y mejoras en la consecución de los objetivos del proyecto, lo cual repercute en la satisfacción de las necesidades de los usuarios de la infraestructura.

38 Equipo BIM Forum Chile (2017) Desarrollo del BIM en Chile: entre el Estado y la Academia. BIM Forum Chile. Disponible en web: http:/ / www.bimforum.cl/2017/12/26/desarrollo-del-bim-enchile-entre-el-estado-y-la-academia/. Consulta realizada el 26 de enero de 2020. 